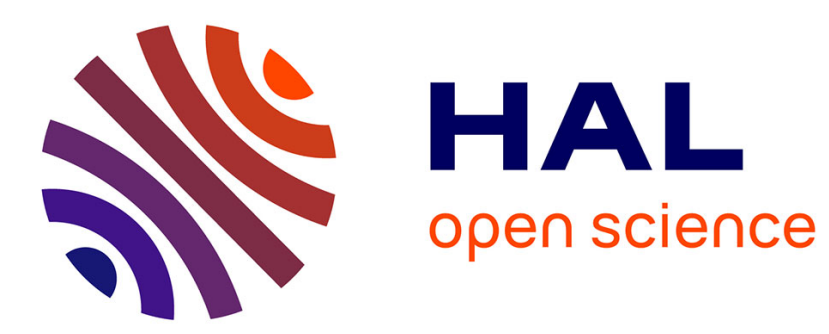

\title{
BPMN Coordination And DEVS Network Architecture For Healthcare Organizations
}

\author{
Mariem Sbayou, Youssef Bouanan, Grégory Zacharewicz, Bruno Vallespir
}

\section{To cite this version:}

Mariem Sbayou, Youssef Bouanan, Grégory Zacharewicz, Bruno Vallespir. BPMN Coordination And DEVS Network Architecture For Healthcare Organizations. International Journal of Privacy and Health Information Management, 2019, 7 (1), pp.103-115. 10.4018/IJPHIM.2019010106 . hal01913718

\section{HAL Id: hal-01913718 \\ https://hal.science/hal-01913718}

Submitted on 7 Dec 2018

HAL is a multi-disciplinary open access archive for the deposit and dissemination of scientific research documents, whether they are published or not. The documents may come from teaching and research institutions in France or abroad, or from public or private research centers.
L'archive ouverte pluridisciplinaire HAL, est destinée au dépôt et à la diffusion de documents scientifiques de niveau recherche, publiés ou non, émanant des établissements d'enseignement et de recherche français ou étrangers, des laboratoires publics ou privés. 


\title{
BPMN Coordination and Devs Network Architecture for Healthcare Organizations
}

\author{
Mariem Sbayou, University of Bordeaux, Bordeaux, France \\ Gregory Zacharewicz, IMT - Mines Alès, France \\ Youssef Bouanan, University of Bordeaux, Bordeaux, France \\ Bruno Vallespir, University of Bordeaux, Bordeaux, France
}

\begin{abstract}
The adoption of business processes (BP) can help healthcare providers in structuring the way information systems and people have to interact. Business process management (BPM) is a methodology that structures a way of representing system processes. At the same time, the human resources are organized in identified or implicit structures that allows individuals to exchange information either related to their work function or not. Nevertheless, the human organizations structure and communication channels are not, up to now, fully captured by the information systems. It may lead to losing part of the useful information exchanged by participants. Accordingly, this article focuses on multi-agent solutions representing social networks in the healthcare domain associated with BPM of patient pathways. The purpose is to study the feasibility of combining BP with agent-based models in order to better improve performance, manage resources, and ensure coordination between them.
\end{abstract}

\section{Keywords}

Business Process Management, Business Process, Healthcare Providers, Multi-Agent, Patient Pathways, Performance, Resources, Social Networks

\section{INTRODUCTION}

Healthcare processes or pathways are typically described informally by text description or by semi-formal languages (Eshuis et al., 2010). A process is generally defined as a sequence of events that uses inputs to produce outputs. Besides, a business process is an activity or set of activities that will accomplish a specific organizational goal. Also, it is considered as a sequence of performed steps that drive information to produce goods and/or provides services. The business process needs to be managed and controlled; it attests the need to use a business process management methodology. According to (Van Der Aalst, Ter Hofstede, and Weske, 2003) business process management (BPM) includes methods, techniques, and tools to support the design, enactment, management, and analysis of operational business processes.

It can be considered as a formalization of classical Workflow Management (WFM) systems and approaches. BPM solutions enable enterprise to measure and standardize processes and also provide 
reusable processes that can be networked. In this context, several languages are used for BPM; the most used one nowadays is Business Process Modeling Notation (BPMN). BPMN has been proposed by the Business Process Modelling Initiative (BPMI) and is currently maintained by the Object Management Group (OMG 2003) that provides this standard for Information Technology (IT) and business actors, it is an increasingly significant standard for process modeling and has received high attention and uptake in BPM practice (Recker 2008). Besides, it is an established standard for business process modelling in industry and economy that is frequently supported by a computer program which enables a quite easy graphical description of complex processes (Scheuerlein et al., 2012).

Furthermore, the analysis of performance of BPMN is crucial, as it helps Business analysts to predict whether the goal can be achieved or not. For this reason, simulation has been identified as a key technique for BP performance analysis (Antonacci et al., 2016). It can be important for business processes as it helps and supports the decision-making process, reduces cost that can occurs in the case wrong decisions haven't been anticipated and as final objective provides a good quality of services.

As argued in (Antonacci et al., 2016), the use of BPM in healthcare sector is becoming a key enabler for the improvement of healthcare processes, since the healthcare environment is becoming more dynamic and volatile, and follows more complex processes, the combination of agent based model with business process may be efficient for resolving simulation's limitations in $\mathrm{BP}$ in terms of resource allocation, in the other hand, managing the availability of resources in healthcare sector is a very challenging problem with very little research attention. For this reason, the use of business process modeling combined with the adoption of simulation-based analysis provides a cost effective, accurate, and rapid way to evaluate alternatives before committing the required effort and resources (Tumay, 1996; Nakatumba, Rozinat, and Russell, 2009).

The points we will explore in this paper are the concept of coupling processes with simulated models and also to propose a viable general approach based on modeling and simulation to address the problem of managing the resources allocation by applying it for healthcare case, so, the general purpose of this paper is to present a new architecture for modeling and simulation that aims to couple tasks followed by actors in the process with agents behaviors described with DEVS (Discrete Event System Specification). Overall, the reminder of this paper is structured as follows first related work and background is presented, then we have the contribution section when we first expose the problem statement, then we give a general overview of the proposed architecture and the methodology, after we will apply the architecture to ensure its feasibility.

\section{RELATED WORK AND BACKGROUND}

BPMN is a graphical notation for drawing business processes (OMG, 2003). It provides a standard notation that is easily understandable by all stakeholders; and also bridges the communication gap that frequently occurs between business process design and implementation. Nowadays BPMN becomes widely used by different organizations, as it is simple to learn, but yet powerful enough to depict the potential complexities of business processes. One of the main complexities of BP is the limitation of its simulation, which is due to the following reasons: lack of simulation know-how of BP analysts, costs and difficulties in retrieving and analyzing the data required for simulation model parameterization, large semantic gap between the business process model and the simulation model and finally, the use of models that may be (partially) incorrect or may not be at the right level of abstraction (Bocciarelli, D'Ambrogio, and Paglia, 2014). In this context, the use of BPMN models in healthcare sector helps to facilitate the management of complex hospital BPs (Antonacci et al., 2016). Accordingly, a lot of researchers in the literature, professionals and health administrators have given various definitions of patient pathways. In (Benabdejlil, 2016), authors define a patient pathway as a description of process's elements of the disease management by the actors of the care network; it corresponds to a trajectory composed of steps that vary not only according to the actors of the network who can interact but also according to the place of residence and the medical history of the patient. 
In the other hand, Multilayer Network has become a vital tool for understanding different relationships and their interactions in a complex system; it is often composed of many single-layer networks with nodes linked within edges. Each layer is sharing the same set of nodes, and edges are divided into two categories: intra-layer networks within each layer and inter-layer edges between vertices of different layers (Murata, 2015). A graph (i.e., a single-layer network) is a tuple $\mathrm{G}=(\mathrm{V}, \mathrm{E})$, where $\mathrm{V}$ is the set of nodes and $\mathrm{E} \mathrm{V} \times \mathrm{V}$ is the set of edges that connect pairs of nodes (Bollobas, 2013). If there is an edge between a pair of nodes, then those nodes are adjacent to each other. This edge is incident to each of the two nodes, and two edges that are incident to the same node are also said to be "incident" to each other (Kivelä et al., 2014). In the field of network science, two types of dynamic are studied: dynamics on networks and dynamics of networks. Dynamics on networks indicates that the activity (or some other property of interest) of nodes changes as a function of time and dynamics of networks indicates that the edges of the network themselves change either in their existence/absence or in their strength (Bassett and Sporns, 2017). Multilayer Networks are useful for complex systems as they allow better understanding system's structure, in addition, many real-world systems do not operate in isolation.

In addition, simulation proved its value in the manufacturing sector and has been used to evaluate process problems in healthcare as well (Harrell and Price, 2000). It has been used for over a decade by health services for improving patient care. In this respect, there are a lot of examples in the literature which provide simulation tools for healthcare organization, in (Laskowski and Mukhi, 2008), authors have developed a tool for managing emergency department, by planning capacity resources utilization and staff capacity. Moreover, authors in (Norouzzadeh et al., 2015) show how modelling and simulation of internal medicine practice process can help on decision making. The results of the simulation, based principally on patient waiting time were able to give an idea about improving resources utilization. Another example is given by Günal and Pidd in (Gehlot, Matthew, and Sloane, 2016) which describes a model of the process flow of patients, that represents the multitasking behavior of medical staff (doctors and nurses), the only issue is that the ignorance of other possible factors such as: doctors' interactions with patients, other medical staff and their working environment lead to incomplete consideration of the problem (Jain et al., 2011).

Moreover, popular approaches include decision analysis, Markov process, mathematical modeling, systems dynamics and discrete event simulation (Fone et al., 2003) (Kanagarajah et al., 2010). The limitation of these approaches is that they ignore the effect of naturalistic human decision-making and behaviors on the performance of healthcare processes. In order to overcome such limitation, the use of Agent Based Modeling ( $\mathrm{ABM}$ ) offers complementary perspectives to model the process of health care domain (Wang, 2009). Agent based modeling (ABM) is formed by a set of autonomous agents that interact with their environment and other agents through a set of internal rules to achieve their objectives (Onggo, 2010) (Grundspenkis and Pozdnyakov, 2006).

In this context, there are some studies In the literature using BPMN model with DEVS model, these studies are based on meta-model approach (OMG 2003), which is one of the most used transformation techniques that includes the mapping of BPMN concepts to DEVS concepts. Based on the proposed approach of BPMN to DEVS (Cetinkaya, Verbraeck, and Seck, 2012) which presented a Model Driven Development (MDD) framework for modeling and Simulation (MDD4MS), and where a set of transformation rules were defined: some BPMN concepts (Pools, Lane, SubProcess) were mapped to DEVS coupled component while task, event (start, end and intermediate) and Gateway were mapped to DEVS atomic component (Bazoun et al., 2016). This proposal doesn't cover the intervention of different resources like (human resources, devices and/or Software services) which may affect the execution of the task. To overcome such limitations, authors in (D'Ambrogio and Zacharewicz, 2016) proposed new approach, by introducing reliability analysis that takes into consideration unexpected failures of the resources that execute the process tasks (unavailability of a resource allocated to task).

Discrete Event system Specification (DEVS) (Zeigler, Praehofer, and Kim, 2000) is formalism for modelling Discrete Events Systems. The hierarchical and modular structure of DEVS allows 
defining multiple models that are coupled to work together in a single and model by connecting their input and output through messages (Wainer, 2009). In the same way, the resulting model can also be coupled with other models defining multiple layers in the hierarchical structure. In DEVS, atomic models define the behaviour of the system, and coupled models describe the structure of the system. The DEVS formalism has several advantages. The reason behind using DEVS is that it is based on dynamical systems theory and provides well defined concepts for coupling components, hierarchical and modular model construction, and an object-oriented substrate supporting repository reuse. Modular construction is one of the most important characteristics of DEVS because it allows the modeler to design and construct each model independently for optimal efficiency. As long as models adhere to certain protocols, they can interact which each other (Pérez et al., 2010). Accordingly, we use VLE (Virtual Laboratory Environment) for the implementation.VLE is an open source software and API under GPL which supports multimodelling and simulation by implementing the DEVS abstract simulator (Quesnel, Duboz, and Ramat, 2009). It integrates specific models developed in most popular programming languages into one single multi- model. It also proposes several simulators for particular formalisms; for example, cellular automata, ordinary differential equations (ODE), differential equations, various finite state automata (Moore, Mealy, Petri-Nets, etc.) and so on. VLE can be used to model, simulate, analyse and visualize dynamics of complex systems. Its main features are: multimodeling abilities (coupling heterogeneous models), a general formal basis for modelling dynamic systems and an associated operational semantic, a modular and hierarchical representation of the structure of coupled models with associated coupling and coordination algorithms, distributed simulations, a component-based development for the acceptance of new visualization tools, storage formats and experimental frame design tools (Bouanan et al., 2015). The purpose of this paper is to present architecture for modeling and simulation in healthcare domain, the proposed architecture aims to overcome BPMN limitations and drawbacks, so for this we propose new approach which includes agent -based technologies. In the coming sections, we give an overview about the architecture, and then we study its feasibility in the case of emergency department (ED).

\section{CONTRIBUTION}

After giving an overview about the issues covered in this paper and before tackling the use case, it is required to introduce the general problem statement of our contribution. In this section, we will provide the problem statement of our study and then explain the proposed architecture and methodology.

\section{Problem Statement}

The use of workflow in healthcare domain is thriving, but since healthcare environment is evolving and complex to manage, it faces some drawbacks including: lack of coordination, limited flexibility during process enactment (Bolcer and Taylor, 1998), inability to cope with dynamic changes in resource levels and task availability, limited ability to predict changes, due to external events, in both the volume and composition of work, lack of performance, scalability and reliability as well (Pang, 2000).

In the other hand, healthcare systems are complex to manage, and they face a lot of problems, poor coordination across healthcare levels is considered to be one of the main obstacles to attaining effective healthcare in many healthcare systems around the world, leading to difficulties in access to care, poor technical quality, discontinuity of care and inefficiencies in the use of resources (Organization and Control, 2008). Patient coordination problem can be insured by a representation of patient pathway where we can clearly see different interactions between resources. In this context, patient pathway has several definitions in the literature, an overall definition is given by ("ARS," 2016), that considers the patient pathway as the comprehensive and continuous care of patients closer to their place of life. It is a question of coordinating the interventions of the 
actors of the prevention and sanitary. The organization on the pathway helps to respond to the growing development of chronic diseases and situations of loss of autonomy that impact the patient's daily life. Patient pathways are based on the intervention of actors: of the health care system (doctors, pharmacists, hospitals, etc.), medical and social services and institutions (for the elderly or disabled), local authorities (town halls), other state services and social protection agencies. It's about organizing a coordinated care: throughout the patient's illnesses a territory and in the proximity of his home.

Overall, a multilayer representation may be helpful for having a meaningful representation of the system under study. Also, for meeting the individual needs of different roles of the patient pathway, BPMN can be considered the most efficient one, since it provides a good understanding of healthcare process (Sang and Zhou, 2015). Besides, Patient pathway can be represented by BPMN as it offers an understandable representation of the system and its interactions, the only issue is that this representation is not dynamic, and as healthcare sector is known as dynamic system that evolve rapidly we propose a new architecture that uses BPMN elements for orchestrating the process where each element has its own behavior described by DEVS model. In order to well describe the architecture, we give a detailed description of the proposed approach and study its feasibility with a small number of data in the case of French healthcare system.

\section{DEVS and BPMN for Healthcare Approach}

In this section we will explain the used approach for modeling and simulation which is applied for healthcare sector, our main objective is to propose a solution for combining BPMN with dynamic models in order to manage availability of resources. For this, we choose a region for the study, specify participants and collect relevant data, then we draw the BPMN of the chosen case study for ensuring coordination and specifying exchanged information between resources. Interactions between entities are divided according to a multilayer network described in (Sbayou et al., 2017).

\section{General Overview of the Architecture}

In Figure 1, we give an overview about the proposed architecture, our aim is to be able to use exchanged information between participants in the BPMN diagram for launching the DEVS network process, for this we extract the XML file of the BPMN model, and we read each of its component, then according to the message flow contained in the xml file, we create an action in the network. In the other hand, participants in the pathway are described as node in the multilayer network and as agents in DEVS model (Sbayou et al., 2017). Besides, healthcare resources' attributes are taken from database that contains information collected from yellow pages, some population's characteristics are generated according to specification given by the National Institute of Statistics and Economic Studies (Ferret, 2017), and geographical positions of patient are generated randomly. The agent-based simulation uses a MySQL database and an XML file generated from BPMN process for inputs. DEVS based simulation for modeling agent's behaviors and R-Studio tool for visualizing results. This approach allows us to study the dynamic part of BPMN model by integrating its elements in the global model that describes resources' behavior, the global DEVS model is a couple model of $\mathrm{xml}$ atomic model and the model that represent integration between participants in the BPMN. We draw an overview about the proposed architecture in Figure 1.

\section{METHODOLOGY}

As healthcare processes are complex to manage and simulate, we chose a category of model that can be used by non-specialist of M\&S, and that is divided into 3 blocks.

The proposed modeling system is described in Figure 2, and it is divided as below: 


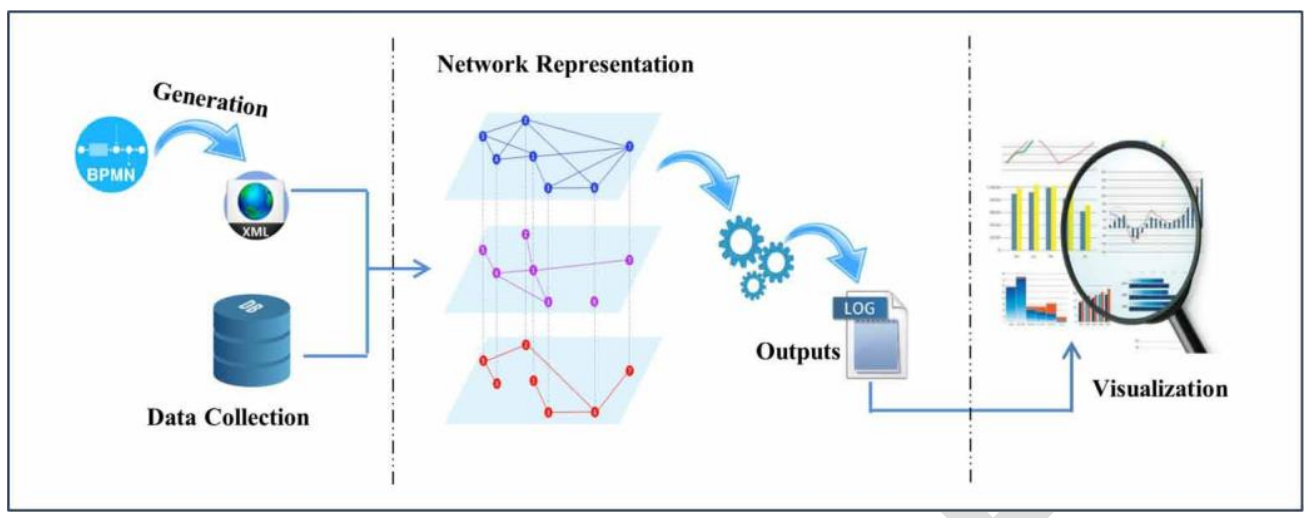

Figure 2. Block diagram for modeling systems

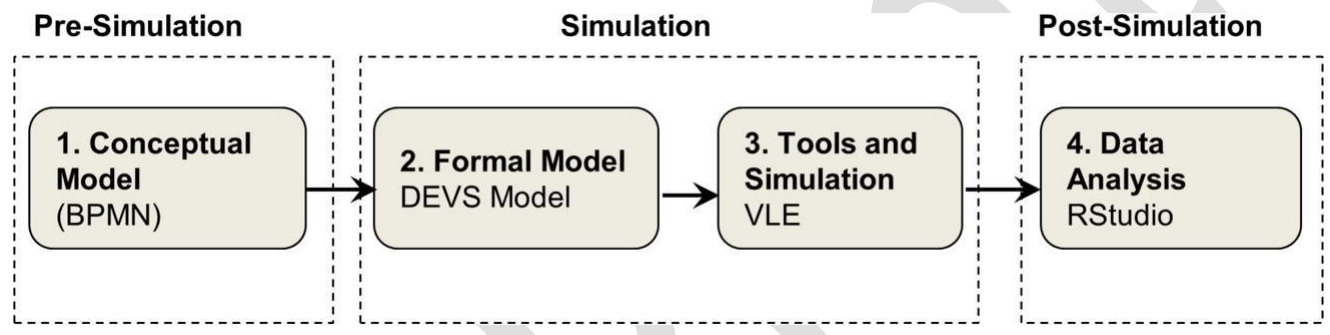

- Pre-Simulation: We define a conceptual model of the studied issue using BPMN. The BPMN process contains pools where each pool describes a participant in the workflow. Also, the structure of the organization of the service is described under a multiplex social network, as described in (Sbayou et al., 2017);

- Simulation: The process starts by transforming the BPMN model to a formal model. Each agent is specified as an atomic model. BPMN model is then specified as a coupled DEVS model. DEVS is used for simulation which is run using VLE;

- Post-Simulation: We use $\mathrm{R}$ to process the results. $\mathrm{R}$ is a programming language for statistical computing and graphics. RStudio tool is used to visualize the simulation results and to conduct analysis.

\section{Operation of the XML Manager}

As mentioned previously, we generate the xml file of the BPMN model for using it on orchestrating connection between patient and healthcare resources, and also it serves as input for simulation, the BPMN is used to ensure coordination between resources at first, and then an.xml file is generated where we will extract tasks that would be able to decide which resources to choose. For this, we create a library of DEVS atomic model, this model will read the xml file where each information is described by tags, the atomic model of the XML controller is describe in (Figure 3), the model has two states, "Init" and "Phase_0", at first, the model send an out information to other model according to the healthcare resources' profession, the output information are responsible for checking the available resources and establish connection between patient and available resources who are going to participate in his pathway. 


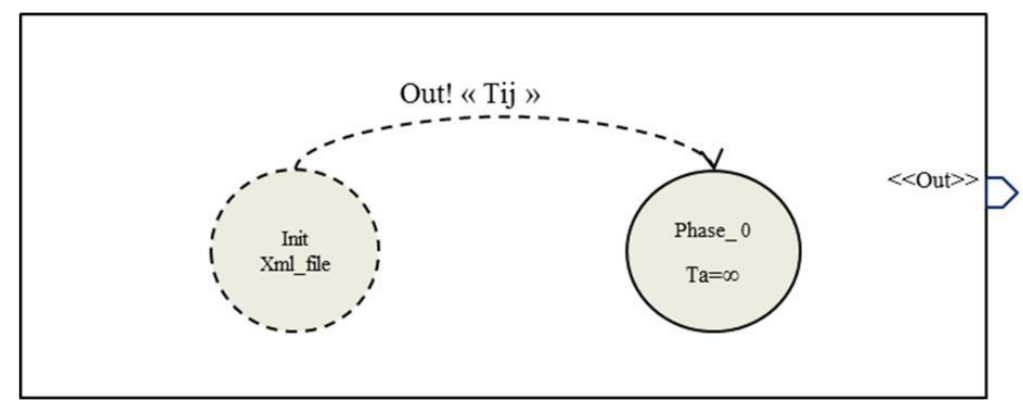

\section{CASE STUDY}

In this section we will study the feasibility of our approach in the case of emergency department for a region located in southwest of France, this application is for proving the connection between actors' tasks that are contained in the BPMN process, and the system's behavior described by DEVS.

\section{Healthcare System in France}

The French healthcare system covers both public and private hospitals, doctors and other medical specialists who provide care services to French resident. It is accessible for all residents, independently of their age, income or status. French resident has to register a General Practitioner "Medecin Traitant", in order to ensure full eligibility to reimbursement of health costs. While following this process, the General Practitioner (GP or G), becomes the principal route which follows the patient care pathways "parcours de soin". In some cases, the patient can directly go to specialists without passing through GP. These specialists are: gynecologists, ophthalmologists, dentists and psychiatrists (for patients under 26 years old). In emergency cases, there is no appointment needed, in addition, emergency departments (ED) are the most complex system in healthcare sector, they offer care for life-threatening and other emergency cases over 24 hours daily, 7 days a week, accordingly, they usually require a lot of resources, at any time. The shortage of resources may lead to the recurrent problems such as overcrowding, congestion and also patient's dissatisfaction. For this reason, we propose to study the feasibility of our system for emergency departments and then apply it for other health cases.

\section{Data Collection}

Accordingly, we have retrieved geographical information about required resources in ED. As the number of existent resources is very important, we have located about 96 resources in a small sub region of "Nouvelle Aquitaine." Generally speaking, we extracted information about: Hospitals (H), Radiologists(R) and General Practitioner (GP and R not needed in the case of ED), we also generated population according to id, age, latitude and longitude parameters. The Figure 4 shows different existent resources, the orange bubbles represent patients with emergency needs, green babbles localize general practitioners, yellow ones are hospitals in the chosen region, and finally red bubbles represent radiologists in the chosen region. The database gives the positioning of the different resources that will be used at simulation time to compute their reachability. Rules of proximity threshold have been defined to be used at simulation time when a resource is reached by the patient himself or through the healthcare social network by the GP or the ED, in the ED, the process doesn't require nor GP nor R, and in emergency cases the patient is supported by ED without any appointment or recommendation. 


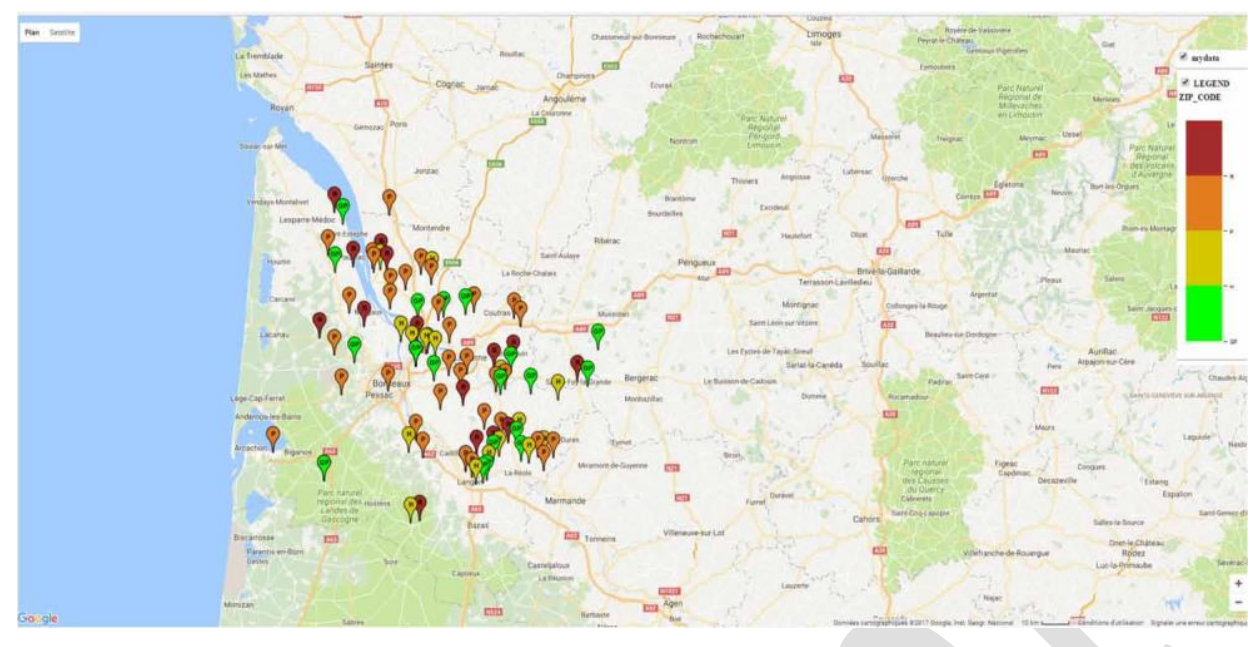

\section{Patient Pathway in ED}

For our case study, we take the example of the University Hospital Center (CHU of Bordeaux). Considering the $\mathrm{CHU}$ of Bordeaux; we have collected some statistical information about the patients as well as their care in the emergency department. The hospital is visited daily by about 149 patients, the completeness of reception is $100 \%$, the sex ratio representing the number of men and number of women at birth during a given age group is $1,17.1 \%$ of patients visiting the hospital are under one year old, $14 \%$ are patients under 18 years of age, patients aged 75 years and over account for $16 \%$, and $7 \%$ of patients are not from the region. The average duration of process for each patient is about 4 hours according to ("ORU," 2017). In order to well understand steps followed by the patient in ED, we drew a BPMN diagram that describes roles of each participant, we can see that we have 2 pools in Figure 5, one for patient and the second one is for emergency department, in the case of ED in CHU of Bordeaux, the principal resources are the secretary, nurses and emergency physicians, hence, once the health issue occurs, the patient selects the GP or ED according to his state, for emergency cases the patient visits the hospital without any appointment, we can see that we have in Figure 4, colored tasks (orange, red and green), this tasks are described by tags information in the $\mathrm{xml}$ file, the orange tasks are called decisional tasks, like the task named "select hospital" in the patient pool, this task is responsible for launching the connection between the patient and the hospital, in the second pool named hospital, we have the secretary who is responsible of registration and providing patient's information to nurses, the available nurse starts the process by the triage of patient, the queuing system follows the first in first out (FIFO) option, red tasks in hospital pool are responsible of changing the state of resources, so, once the nurse is in red tasks he will be busy and not able to work with another patient, the nurse has to select the available physician that defines the priority of patients: resuscitation, treatment that lead to hospitalization, or examination which may be followed by hospitalization or redirection to home, in the physician lane we have green task named redirect home that means that resources are free and can be used by the next patient.

\section{RESULTS AND DISCUSSION}

In order to apply the architecture, we take the example of 10 patients who arrived to hospitals. First we extract the nearest hospital (proximity rule) to each one, when the hospital receives patients, and after registration, the process of checking of available resources is launched according to availability 


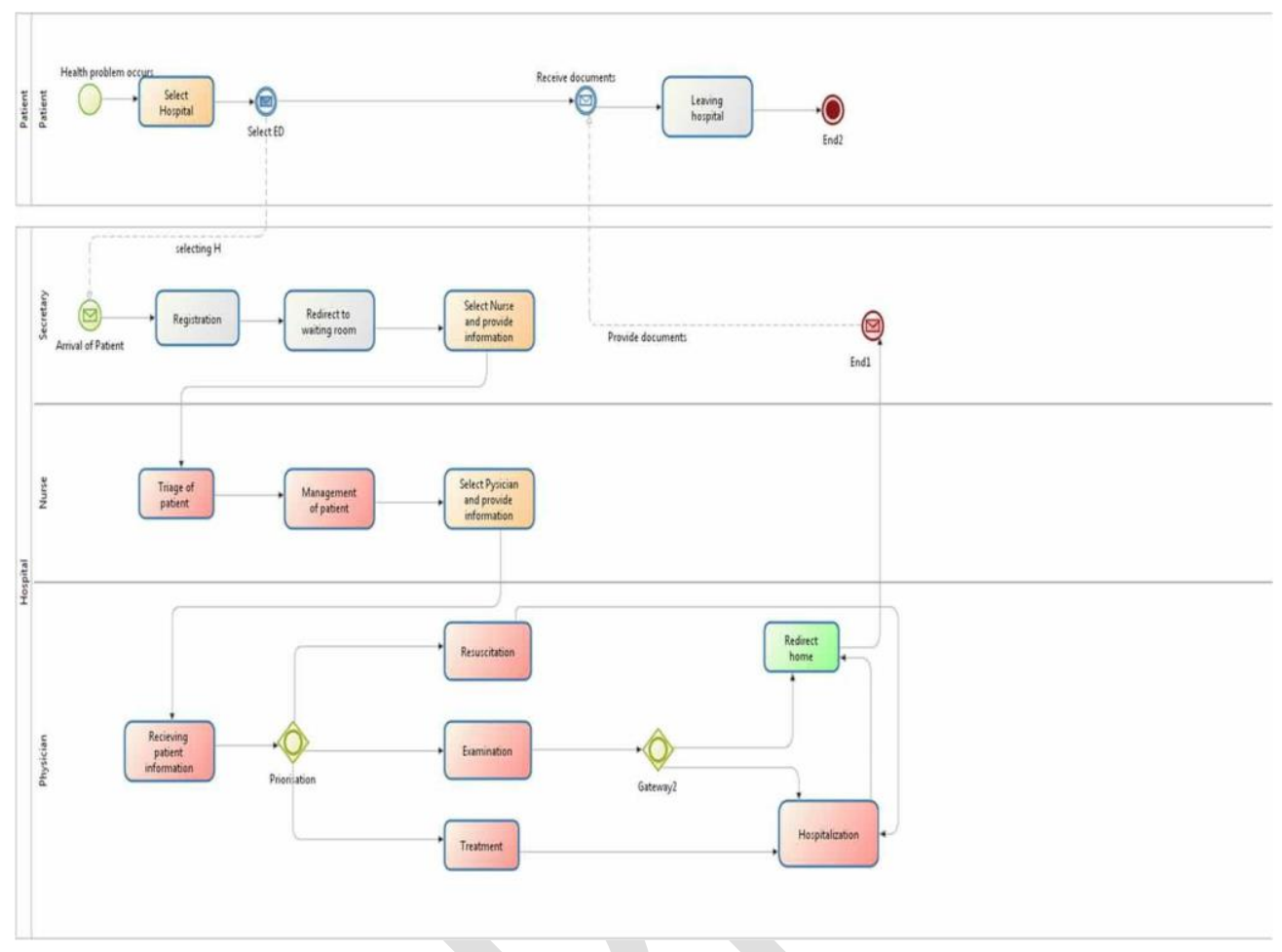

rule. The basic simulation has permitted to identify and select resources to support the execution of the BPMN model for patients (Pi). In detail, we supposed that only 1 administrative agent, 3 nurses and 4 emergency physicians are available at specific time, the Figure 6 shows simulated DEVS network of one agent (A) connected to several resources (patients (Pi), nurses (Ni) and physicians (Di)). It details all connected resources in the case of arrival of 10 patients at a specific time. We can observe

Figure 6. resource network of available resources

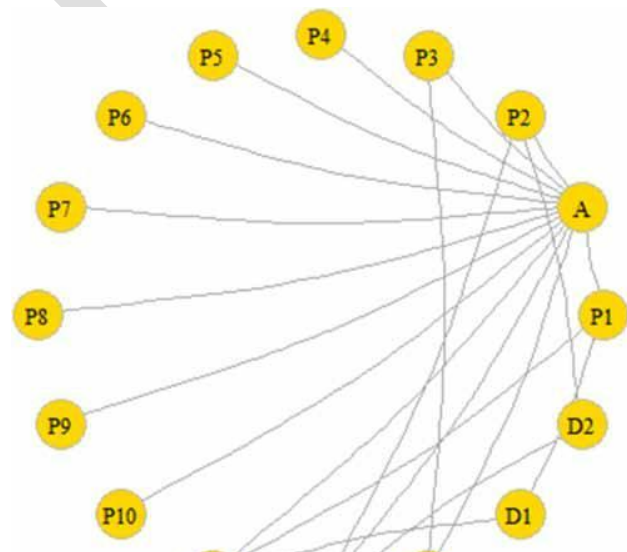


that some patients are only registered because there are not enough health resources which may take them in charge. We can also observe that that administrative agent is connected to all coming patients that are still waiting for their turn in order to join the network.

This study ensures the feasibility of connecting BPMN and DEVS model for sample simulation of the allocation of available resources, the main idea is to test and verify the proposed architecture, then to apply the architecture for a big system with a lot of exchanged information and a complex queuing system.

\section{CONCLUSION AND PERSPECTIVES}

This paper presents a new architecture based on business process and multidimensional network for modeling in healthcare sector. It uses BPMN to define the patient pathway process connected with an ABM network. It defines and run the search for resources and their allocation based on a multi-layer different healthcare stakeholders' networks exploration. The objective is to study the impact of dynamic allocation of participant actors in BPMN healthcare pathways. Such an approach can be proposed to study a territory in terms of sufficient or insufficient resources available in a specific area. The case study, located in Bordeaux city, has been possible thanks to data coming from yellow pages that contains information about healthcare resources with their geographic location. The presented case study aims to manage resources and shows their impact on the performance of the process. As future work, we will include progressively new parameters as new resources (equipment), as well as more demographic information of patients and staff, budget constraints in order to manage healthcare system. We also propose to observe the impact of giving new geographical positions for healthcare resources, as well as to propose the implementation of new services, and run different scenarios to see how the system could be more performant. Finally, we want to apply our architecture in other sectors in order to make it functional for different application domain. 


\section{REFERENCES}

Antonacci, G., Calabrese, A., D’Ambrogio, A., Giglio, A., Intrigila, B., \& Levialdi Ghiron, N. (2016). A BPMN-Based Automated Approach for the Analysis of Healthcare Processes. In 2016 IEEE 25th International Conference On Enabling Technologies: Infrastructure for Collaborative Enterprises (WETICE) (pp. 124-129). IEEE. Retrieved from http://ieeexplore.ieee.org/abstract/ document/7536444/

ARS. (2016). Parcours de soins. Retrieved from https://www.ars.sante.fr/sites/default/files/2017-01/2016-0111_lexique_VF_0.pdf

Bassett, D. S., \& Sporns, O. (2017). Network Neuroscience. Nature Neuroscience, 20(3), 353-364. doi:10.1038/ nn.4502 PMID:28230844

Bazoun, H. et al.. (2016). SLMToolBox: Enterprise service process modelling and simulation by coupling DEVS and services workflow. IJSPM, 11, 453-467.

Benabdejlil, H. (2016). Modélisation Des Processus de Soins: Vers Une Implantation de Nouveaux Services à Valeur Ajoutée.

Bocciarelli, P., D’Ambrogio, A., \& Paglia, E. (2014). A Language for Enabling Model-Driven Analysis of Business Processes. In 2014 2nd International Conference On Model -Driven Engineering and Software Development (MODELSWARD) (pp. 325-332). IEEE. Retrieved from http://ieeexplore.ieee.org/abstract/ document/7018480/

Bolcer, G. A., \& Taylor, R. N. (1998). Advanced Workflow Management Technologies, Software Process Improvement and Practice, 4(3), 125-171. doi:10.1002/(SICI)1099-1670(199809)4:3<125::AIDSPIP100>3.0.CO;2-J

Bollobas, B. (2013). Modern Graph Theory. Springer Science \& Business Media.

Bouanan, Y., Forestier, M., Ribault, J., Zacharewicz, G., Vallespir, B., \& Moalla, N. (2015) . Simulating Information Diffusion in a Multidimensional Social Network Using the DEVS Formalism (WIP). In Proceedings of the Symposium on Theory of Modeling \& Simulation: DEVS Integrative M\&S Symposium (pp. 63-68). Society for Computer Simulation International. http://dl.acm.org/citation.cfm?id=2872974

D'Ambrogio, A., \& Zacharewicz, G. (2016). Resource-Based Modeling and Simulation of Business Processes. In Proceedings of the Summer Computer Simulation Conference SCSC '16. San Diego, CA: Society for Computer Simulation International. Retrieved from http://dl.acm.org/citation.cfm?id=3015574.3015637

Eshuis, P., ten Pas Sopheon, E., Rutten Sopheon, H., \& Verlinden Zorggemak, J.-M. (2010). State of the Art Clinical Pathway Definition: Gap Analysis. Retrieved from https://itea3.org/project/workpackage/document/ download/249/07011-Edafmis-WP-1-D11.pdf

Ferret, J.-P. (2017). 5879144 Habitants En Nouvelle-Aquitaine Au 1er Janvier 2014.

Fone, D., Hollinghurst, S., Temple, M., Round, A., Lester, N., Weightman, A., \& Palmer, S. et al. (2003). Systematic Review of the Use and Value of Computer Simulation Modelling in Population Health and Health Care Delivery. Journal of Public Health, 25(4), 325-335. doi:10.1093/pubmed/ fdg075 PMID:14747592

Gehlot, V., Matthew, L., \& Sloane, E. B. (2016). From Data to Processes??? Use of Modeling and Simulation in Healthcare. In 2016 IEEE-EMBS International Conference on Biomedical and Health Informatics (BHI) (pp. 320-323). IEEE. http://ieeexplore.ieee.org/xpls/abs_all.jsp?arnumber=7455899

Grundspenkis, J., \& Pozdnyakov, D. (2006). An Overview of the Agent Based Systems for the Business Process Management. In Proc. Int'l Conf. Computer Systems and Technologies.

Kanagarajah, A. K., Lindsay, P., Miller, A., \& Parker, D. (2010). An Exploration into the Uses of Agent-Based Modeling to Improve Quality of Healthcare. In Unifying Themes in Complex Systems (pp. 471-478). Springer.

Kivelä, M., Arenas, A., Barthelemy, M., Gleeson, J. P., Moreno, Y., \& Porter, M. A. (2014). Multilayer Networks. Journal of Complex Networks, 2(3), 203-271. doi:10.1093/comnet/cnu016 
Laskowski, M., \& Mukhi, S. (2008) . Agent-Based Simulation of Emergency Departments with Patient Diversion. In International Conference on Electronic Healthcare (pp. 25-37). Springer. http://link.springer.com/ chapter/10.1007/978-3-642-00413-1_4

Murata, T. (2015). Comparison of Inter-Layer Couplings of Multilayer Networks. In 2015 11th International Conference on Signal-Image Technology Internet-Based Systems (SITIS) (pp. 448-452). doi:<ALIGNMENT. qj $></$ ALIGNMENT > 10.1109/SITIS.2015.122

Nakatumba, J., Rozinat, A., \& Russell, N. (2009). Business Process Simulation: How to Get It Right. In International Handbook on Business Process Management. Citeseer. Retrieved from http://citeseerx.ist.psu. edu/viewdoc/summary?doi=10.1.1.668.3489

Norouzzadeh, S., Riebling, N., Carter, L., Conigliaro, J., \& Doerfler, M. E. (2015). Simulation Modeling to Optimize Healthcare Delivery in an Outpatient Clinic. In 2015 Winter Simulation Conference (WSC) (pp. 1355-1366). IEEE. http://ieeexplore.ieee.org/xpls/abs_all.jsp?arnumber=7408259

OMG. (2003). MDA Guide Version 1.0.1 J. Miller and J. Mukerji, Eds.). Retrieved from http://www.omg.org/ cgi-bin/doc?omg/03-06-01.pdf

Onggo, B.S.S. (2010). Running Agent-Based Models on a Discrete-Event Simulator. In Proceedings of the 24th European Simulation and Modelling Conference (pp. 51-55). Retrieved from https://pdfs.semanticscholar. org/593a/b9dd828bcefb684f6ac9cb56b992077935f9.pdf

Organization, World Health, and Research for International Tobacco Control. (2008). WHO Report on the Global Tobacco Epidemic, 2008: The MPOWER Package. World Health Organization.

ORU. (2017). Panorama Oru Na Web. Retrieved from http://www.orulim.com/fichiers/PANORAMA_ORU_ NA_WEB_2015.pdf

Pang, G. (2000). Implementation of an Agent-Based Business Process. University of Zurich.

Pérez, E ., Ntaimo, L., Bailey, C., \& McCor mack, P. (2010). Modeling and Simulation of Nuclear Medicine Patient Ser vice Management in DEVS. Simulation, 86(8-9), 481-501. doi:10.1177/0037549709358294

Quesnel, G., Duboz, R., \& Ramat, É. (2009). The Virtual Laboratory Environment-An Operational Framework for Multi-Modelling, Simulation and Analysis of Complex Dynamical Systems. Simulation Modelling Practice and Theory, 17(4), 641-653. doi:10.1016/j.simpat.2008.11.003

Recker, J. C. (2008). BPMN Modeling-who, Where, How and Why. BPTrends, 5(3), 1-8.

Sang, K. S., \& Zhou, B. (2015). BPMN Security Extensions for Healthcare Process. In 2015 IEEE International Conference On Computer and Information Technology; Ubiquitous Computing and Communications; Dependable, Autonomic and Secure Computing; Pervasive Intelligence and Computing (CIT/IUCC/DASC/PICOM) (pp. 2340-2345). IEEE. doi:10.1109/CIT/IUCC/DASC/ PICOM.2015.346

Sbayou, M., Bouanan, Y., Zacharewicz, G., Ribault, J., \& François, J. (2017, April). DEVS modelling and simulation for healthcare process application for hospital emergency department. In Proceedings of the 50th Annual Simulation Symposium (p. 4). Society for Computer Simulation International.

Scheuerlein, H., Rauchfuss, F., Dittmar, Y., Molle, R., Lehmann, T., Pienkos, N., \& Settmacher, U. (2012). New Methods for Clinical Pathways - business Process Modeling Notation (BPMN) and Tangible Business Process Modeling (t. BPM). Langenbeck's Archives of Surgery, 397(5), 755-761. doi:10.1007/s00423-0120914-z PMID:22362053

Tumay, K. (1996). Business Process Simulation. In Proceedings of the 28th Conference on Winter Simulation (pp. 93-98). IEEE Computer Society. Retrieved from http://dl.acm.org/citation.cfm?id=256581

Van Der Aalst, W. M., Ter Hofstede, A. H., \& Weske, M. (2003, June). Business process management: A survey. In International conference on business process management (pp. 1-12). Springer. Retrieved from http://link. springer.com/chapter/10.1007/3-540-44895-0_1 
Wainer, G. A. (2009). Discrete-Event Modeling and Simulation: A Practitioner's Approach. C RC Press

Wang, L. (2009). An Agent-Based Simulation for Workflow in Emergency Department. In Systems and Information Engineering Design Symposium SIEDS'09 (pp. 19-23). IEEE. doi:10.1109/SIEDS.2009.5166148

Zeigler, B. P., Praehofer, H., \& Kim, T. G. 2000. Theory of Modeling and Simulation: Integrating Discrete Event and Continuous Complex Dynamic Systems. Academic press

Mariem Sbayou is a Ph.D. student at the IMS Laboratory of University of Bordeaux. Her research domain includes DEVS, Healthcare M\&S. She received her engineering degree as software engineer from the International Academy of Civil Aviation in Morocco.

Gregory Zacharewicz is an Full Professor at the Institute of Mines and Telecom (IMT - Mines Ales) with both competences in enterprise engineering and M\&S.

Youssef Bouanan is a Data Scientist in the industry. He received his Ph.D. degree in Production Engineering from University of Bordeaux, France. His research interests include modeling and simulation theory, social network and workflow.

Bruno Vallespir is a full professor at University of Bordeaux, IMS laboratory. He is a member of several international working groups (IFIP, IFAC); he participated in 5 European projects, directed more than 20 PhD students, and has written more than 120 papers for journals and conferences. 